Ann. Zootech., xg68, 17 (I), 7 I-75.

\title{
VARIATION DE LA TENEUR EN HYDROXYPROLINE DE MUSCLES DE PORCS LARGE WHITE ET PIÉTRAIN
}

\author{
R. BOCCARD \\ avec la collaboration technique de A. TaLmant et Colette Girard \\ Laboratoire de Recherches sur la Viande, \\ Centre national de Recherches zootechniques, 78 -Jouy-en-Josas \\ Institut national de la Recherche agronomique
}

SOMMAIRE

Dans 7 muscles de 5 porcs de race Large White et de 5 porcs de race Piétrain, les teneurs en hydroxy. proline exprimées par le rapport de l'azote de cet acide aminé à l'azote total ont été comparées.

Les teneurs sont significativement différentes entre muscles. I.a différence raciale est également hautement significative. Les muscles étudiés des pores de race Piétrain contiennent de 25 à $30 \mathrm{p}$. Ioo d'hydroxyproline de moins que les muscles correspondants des pores de la race Large White.

Les conséquences de ces variations sur le plan de l'élevage et la qualité des viandes sont discutées en vue d'une amélioration de ce caractère.

\section{INTRODUCTION}

Les qualités organoleptiques et technologiques de la viande sont largement conditionnées par sa composition chimique. La teneur en tissus conjonctifs, en particulier, influence largement la tendreté et la valeur nutritive. Les variations quantitatives de ces tissus sont généralement étudiées dans les différents muscles des animaux de boucherie, en prenant essentiellement en considération les protéines fibreuses qui les constituent : 1'élastine et surtout le collagène dont la part est prépondérante. En général, celle-ci est mesurée par la détermination de 1'hydroxyproline, acide aminé spécifique de cette protéine musculaire.

I1 est maintenant bien établi que la teneur des muscles en collagène varie en fonction de nombreux facteurs : nature du muscle, âge et état physiologique des ani- 
maux. L'influence de l'hérédité, par contre, a été moins étudiée, bien que de nombreuses observations pratiques, soulignant les écarts de tendreté entre sujets de même poids et de même âge, laissent supposer une influence génétique qui pourrait s'exprimer par des différences dans la synthèse des protéines.

Nous rapportons ici les résultats d'une série de déterminations des quantités d'hydroxyproline de divers muscles de porcs de race Large White et Piétrain.

\section{MATÉRIEI, ET MÉTHODES}

Pour chacune des races Large White et Piétrain sur 5 porcs mâles castrés de Ioo kg vif environ, tout ou partie des muscles suivants furent prélevés le lendemain de l'abattage :

$$
\begin{aligned}
& \text { Muscles entiers }\left\{\begin{array}{l}
\text { Semi tendinosus, } \\
\text { Semi membranosus, } \\
\text { Adductor, } \\
\text { Biceps brachii, } \\
\text { Triceps brachii caput laterale, }
\end{array}\right. \\
& \text { Parties de muscle }\left\{\begin{array}{l}
\text { Pectoralis profondus, } \\
\text { Latissimus dorsi. }
\end{array}\right.
\end{aligned}
$$

Les muscles, débarrassés de leur graisse, ont été broyés, homogénéisés, puis une fraction aliquote lyophilisée. La matière sèche obtenue a été pulvérisée au broyeur à billes jusqu'à ce que tout. l'échantillon passe au travers d'un tamis aux mailles de $0,4 \mathrm{~mm}$. Sur la poudre homogénéisée ont été prélevés 4 échantillons de $500 \mathrm{mg}$ pour la détermination de l'hydroxyproline et 3 échantillons de roo $\mathrm{mg}$ pour celle de l'azote total.

L'hydroxyproline a été déterminée par la méthode colorimétrique de BERgmanN et Loxley (I960), adaptée à l'auto-analyseur Technicon. Les échantillons étaient hydrolysés en présence d'HCl $6 \mathrm{~N}$ sous reflux pendant 8 heures, passés sur charbon, concentrés, puis neutralisés avant la réaction colorimétrique. L'azote total a été déterminé après minéralisation sulfurique par la méthode colorimétrique de Thomas-Berthelot, adaptée à l'auto-analyseur selon un montage inspiré de celui de FerRari (Ig60).

\section{RÉSULTATS}

Pour éliminer les causes de variation, celles dues en particulier aux différences d'état d'engraissement, la comparaison entre muscles, animaux et races fut faite d'après l'importance de l'azote de 1'hydroxyproline par rapport à l'azote total. La teneur en $\mathrm{N}$ de 1'hydroxyproline fut calculée selon le rapport théorique de l'azote $\mathrm{N}$ dans la $\mathrm{L}$ hydroxyproline, soit:

$$
\frac{\mathrm{I} 4,0067}{\mathrm{I} 3 \mathrm{I}, \mathrm{I} 3}=0, \mathrm{I} 068 \mathrm{I} 5
$$

Les résultats sont résumés dans la figure I qui compare par race les résultats moyens obtenus pour chacun des muscles.

La teneur en hydroxyproline exprimée selon le rapport : $\frac{\mathrm{N} \text { Hypro }}{\mathrm{N} \text { total }}$ est différente pour chaque muscle des deux races. Ia différence s'accroît en fonction de leur teneur en hydroxyproline. 
L'analyse de variance (tab1. I) démontre une interaction race-muscle hautement significative. Les différences raciales sont donc plus ou moins importantes selon le muscle. Cependant, sur les 7 muscles considérés, la différence moyenne entre les deux races est hautement significative.

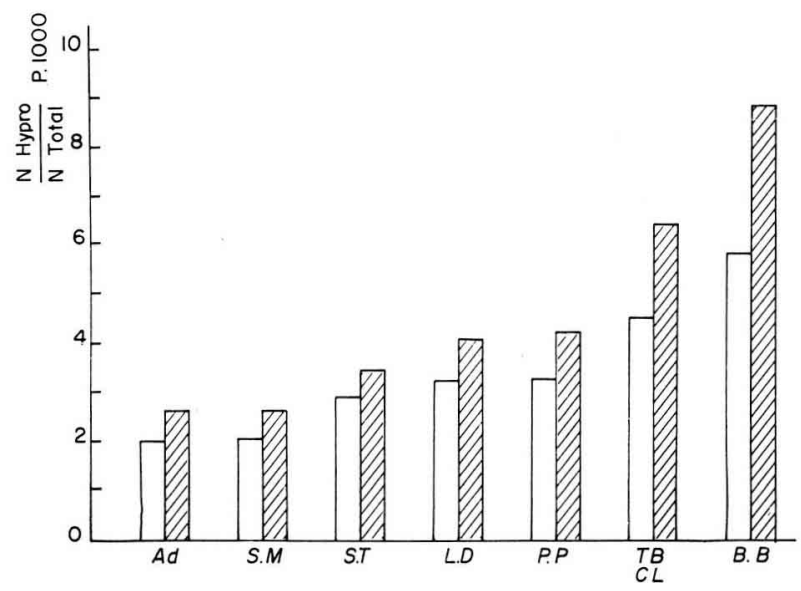

Figure I

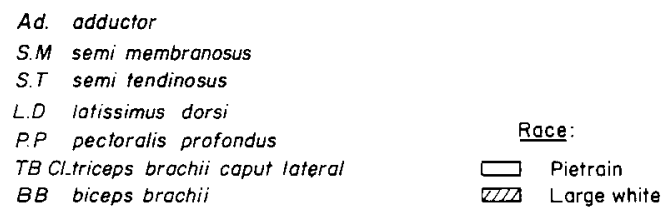

TABI.EAU I

\begin{tabular}{|c|c|c|}
\hline & Dectrés de liberté & Carrés moyens \\
\hline 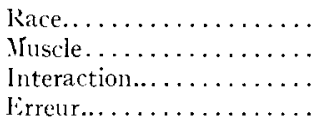 & $\begin{array}{c}1 \\
6 \\
6 j \\
55^{*}\end{array}$ & $\begin{array}{l}25,72 * * \\
32,26 * * \\
2,11 * * \\
0,11\end{array}$ \\
\hline $\begin{array}{l}\text { * Une domée manqu } \\
\text { * Test F significati }\end{array}$ & $\begin{array}{l}\text { te a été estimée. } \\
\text { i seuil } 1 \text { p. 10o. }\end{array}$ & \\
\hline
\end{tabular}

\section{DISCUSSION}

I. I a teneur en hydroxyproline est une mesure très généralement adoptée pour évaluer la quantité de collagène présent dans la viande. Le mode d'estimation de ce dernier change cependant avec les auteurs selon qu'ils retiennent un " collagène type " renfermant plus ou moins d'hydroxyproline. Si sa teneur varie de 12,5 à $I_{4}, 4 \mathrm{p}$. Ioo, le coefficient de transformation pour estimer la quantité de collagène à 
partir de l'hydroxyproline passe de 8 à 7 , I. Dans notre comparaison, plutôt que d'utiliser un coefficient approché pour calculer la teneur en collagène, nous avons préféré traduire les teneurs en hydroxyproline par les quantités d'azote $(\mathrm{N})$ de cet acide aminé. Nous avons également préféré rapporter cet azote de l'hydroxyproline à l'azote total plutôt qu'aux protéines totales.

De nombreux auteurs, cependant, ont utilisé à la suite de Mol,Her et AnToNAcopoulos (r957) la formule:

$$
\frac{\text { Hypro } \times 8 \times \text { Ioo }}{\text { Azote total } \times 6,25}
$$

PRÄNDL, HAAs et MOLKE, (I967) ont discuté la valeur de ce mode de calcul. Ils ont montré que le choix des deux coefficients, d'une part 8 pour transformer l'hydroxyproline en collagène et 6,25, d'autre part, pour calculer la quantité d'azote protéique à partir de l'azote déterminé par la méthode Kjeldalh, pouvait conduire pour les viandes à des pourcentages de collagène erronés. Les erreurs, variables avec la teneur de l'échantillon en hydroxyproline, peuvent, selon ce calcul, atteindre jusqu'à I 2 points.

2. Les valeurs des teneurs en azote de l'hydroxyproline par rapport à l'azote total que nous avons obtenues sont très variables entre les muscles et selon les races. Elles sont sensiblement plus fortes que celles obtenues sur des muscles par Pujszo (I966), (0,95, I,78 et 3,46 respectivement pour le Psoas major, le Semi membranosus et le Triceps brachii), mais moindres que celles rapportées par LINKE et al. (Ig67) pour des morceaux de boucherie ou des parties de carcasses. Ces différences semblent pouvoir être expliquées par le mode d'échantillonnage. La teneur en collagène pouvant fortement varier selon le degré de parage du muscle et, dans les morceaux, selon l'importance des parties tendineuses et aponévrotiques.

3. Les différences de teneur sont d'autant plus fortes que l'on considère des muscles plus riches en tissu conjonctif. La différence entre le même muscle de chacune des deux races est ainsi plus marquée pour les muscles Biceps brachii et Triceps brachii caput laterale, que pour les muscles Semi tendinosus et Semi membranosus.

Cette différence raciale importante est à rapprocher de celle observée entre les animaux normaux et les animaux présentant une hypertrophie musculaire dans certaines races bovines (race Charollaise : Boccard, Dumon't et Schmitt (I967) et race Frisonne : Lawrie, Pomeroy et Wilimams (I963). Chez les sujets hypertrophiés, la teneur en hydroxyproline des muscles est plus faible.

Par ailleurs, les pores de Piétrain offrent, par rapport aux porcs Large White, un pourcentage plus élevé de muscles, en particulier dans leurs membres postérieurs (Mesle, Giron, Dumont, I959). Ce point, ajouté à leur conformation plus particulièrement rebondie, peut les faire considérer comme des animaux atteints d'hypertrophie musculaire ou, selon le vocabulaire en usage dans l'élevage, comme des " culs de poulain " ou " culards ». Ce caractère héréditaire pourrait être monofactoriel (Ollivier et Lauvergne, ig67).

4. Sur le plan de l'élevage et de l'amélioration des animaux, la variabilité de la teneur en hydroxyproline que nous avons constatée offre des possibilités d'action par un meilleur choix des reproducteurs ou par croisement, une fois précisée la teneur souhaitable en collagène. 
Sur le plan organoleptique et nutritionnel, il paraît utile de rechercher de faibles teneurs en tissus conjonctifs dans les muscles. Mais il ne faudrait pas que la sélection, continue dans ce sens, entraîne des troubles dans d'autres organes, compromettant de ce fait les capacités de reproduction ou la rusticité des animaux, notamment par l'insuffisance du squelette par rapport à la musculature (BOCCARD et DuMONT, I967).

De plus, sur le plan de la technologie de la viande, les fabrications nécessitent, pour leur cohésion, des proportions définies des différentes protéines et vraisemblablement un taux minimum de collagène générateur de gélatine.

Un programme de modification par la sélection du taux de collagène musculaire, bien que possible à envisager, devrait être, au préalable, appuyé sur les résultats d'étude des caractères zootechniques des animaux et de la valeur des produits de fabrication.

Reçu pour publication en janvier 1968.

\section{SUMMARY}

VARIATIONS IN THE RATE OF HYDROXYPROLINE IN MUSCLES

OF " LARGE WHITE " AND " PIÉTRAIN " PIGS

The rates of hydroxyproline in 7 muscles of 5 Large White and 5 Piétrain pigs were expressed as nitrogen of this aminoacid per total nitrogen and compared.

The differences between the 7 muscles of each animal were significant.

The difference between the two breeds was highly significant : the Pietrain muscles contained 25 or 30 per cent less hydroxyproline than the Large White.

The possible improvements in breeding and meat quality that can be infered from these variations are discussed.

\section{RÉFÉRENCES BIBLIOGRAPHIQUES}

Bergman I., Loxley R., I963. Two improved and simplified methods for the spectrophotometric determination of hydroxyproline. Anal. Chem., 35, I961-1965.

Boccard R., Dumont B. I., Schmitt O., I967. Note sur les relations entre la dureté de la viande et les principales caractéristiques du tissu conjonctif. 13th Meat Research Workers Meet. Rotterdam, I967 (23 p.)

Duyont B. L., Boccard R., ig67. Le rapport muscles/os, critère de sélection des bovins de boucherie II $I^{\mathrm{e}}$ Symp. Intem. Zoolech., Milan, 1967 (4 p.).

Ferrari A., 1960. Nitrogen determination by a continuous digestion and analysis system. Ann. N. Y. Acad. Sci., 8\%, $792-800$.

Lafrie R. A., Pomeroy R. W., Williams D. R., i 964 . Studies in the muscles of meat animals. IV. Comparative composition of muscles from Doppellender and normal sibling. J. agric., Sci., 62, 89-92.

Linke H., Fleischmann O., Woltersdorf W., Wirti F., ig67. Der Bindegewebegehalt in Schlacht tierkörpern von Schwein und Rind. III. Mitteilung : Zum Begriff "Fleisch wie gewachsen ". Fleischwirtschaft, 47, 744-754.

Mesle L., Girox J., Dumont B. L., i959. Anatomie et composition chimique du jambon. 5th Meat Research Workers Meel. Paris, I959 (I 5 p.).

Molher K., Antoxacopoulos M., I957. Chemische Bestimmung von Bindegewebe in Fleisch und seinen Zubereitungen. Z. Lebensmittel. Unters. U. Forsch., 106, 425-440.

Olimier L., Lalvergne J. J., i 967 . Étude du déterminisme héréditaire de l'hypertrophie musculaire du porc de Piétrain. Ann. Med. Vel., 111 ro4-109.

Pränd O., HaAs J., POLKE E., I967. Zur Differenzierung des Rohproteins in Fleischwaren anhand der Hydroxyprolinmethode zum Zwecke der Qualitätsbeurteilung. Fleischwirtschaft, 47, $5^{81} 5^{86 .}$

Pujszo K., 1966. Investigation into meat connective tissue in pigs. I. Hydroxyproline content in some pig muscles (en polonais). Rocsnicki Nauk Rolniczych, 89, B 1, 61-66. 